\title{
Intensive calcium monitoring improves outcomes on hungry bone syndrome in hyperparathyroidism
}

\author{
Joana Lima Ferreira ${ }^{1, \#}$, Francisca de Brito Marques ${ }^{1, \#}$, Ligia Freire ${ }^{2}$, Virginia Soares ${ }^{2}$, \\ Luisa Guerreiro ${ }^{3}$, Sandra Silva ${ }^{4}$, Carolina Guedes ${ }^{3}$
}

${ }^{1}$ Department of Endocrinology, Hospital Pedro Hispano, Matosinhos Local Health Unit, Matosinhos, Portugal; ${ }^{2}$ Department of Surgery, Hospital Pedro Hispano, Matosinhos Local Health Unit, Matosinhos, Portugal; ${ }^{3}$ Intermediate Care Unit, Department of Internal Medicine, Hospital Pedro Hispano, Matosinhos Local Health Unit, Matosinhos, Portugal; ${ }^{4}$ Department of Nephrology, Hospital Pedro Hispano, Matosinhos Local Health Unit, Matosinhos, Portugal ${ }^{*}$ Contributed equally to this work.

E-mail: joanalferreira@gmail.com

Objectives. Hungry bone syndrome (HBS) is a severe and underdiagnosed complication of parathyroidectomy in the treatment of primary hyperparathyroidism (PHP) and secondary hyperparathyroidism to chronic kidney disease (SHP-CKD).

Methods. A longitudinal study was conducted to compare the postoperative outcomes of patients who developed HBS in two different time frames: before and after implementing a protocol with an intensive electrolytic monitoring and an algorithm regarding electrolytic supplementation.

Results. Overall, 77 parathyroidectomies were included. In PHP, a protocol implementation led to an increased admission of patients in the Intermediate Care Unit for intensive electrolytic monitoring $(\mathrm{p}<0.001)$ and an increased rate of oral calcium replacement during hospital stay $(\mathrm{p}=0.013)$ compared to pre-protocol era. In SHP-CKD, duration of intravenous calcium replacement was reduced $(\mathrm{p}=0.010)$. The prevalence of HBS (9.8\% in PHP and 58.3\% in SHP-CKD) was similar between the two periods, although its diagnosis had an increased trend in PHP since the protocol implementation. None of the diagnosis of HBS was established due to hypocalcemic symptoms in the post-protocol era (contrary to pre-protocol period, $\mathrm{p}=0.021$ ). Both hypocalcemia length and duration of surgical ward hospitalization were reduced ( $\mathrm{p}=0.047$ and $\mathrm{p}=0.042$, respectively).

Conclusions. An improved assessment of hyperparathyroidism and a decrease in HBS severity were noted in the post-protocol era. We strongly recommend the implementation of a standardized protocol with an intensive phosphocalcium monitoring in the high-risk patients who undergo parathyroidectomy due to hyperparathyroidism as it improves the health care and management of HBS.

Key words: hypocalcemia, hungry bone syndrome, hyperparathyroidism, parathyroidectomy, calcium, bone

Hungry bone syndrome (HBS) is a challenging and often underdiagnosed clinical entity that may complicate the postoperative management of parathyroidectomy for primary hyperparathyroidism (PHP) and secondary hyperparathyroidism (SHP) to chronic kidney disease (CKD). Literature data regarding its definition, prevalence, risk factors, clinical course, and perioperative management are still limited and controversial (Goldfarb et al. 2012; Witteveen et al. 2013; Ho et al. 2017; Cartwright and Anastasopoulou 2020).

There are only a few reported cases of HBS in literature and epidemiological data regarding its incidence is scarce and heterogeneous (Witteveen et al. 2013;

Corresponding author: J. Lima Ferreira, Servico de Endocrinologia, Departamento de Medicina, Hospital Pedro Hispano, Rua Dr. Eduardo Torres, 4464-513 Senhora da Hora, Matosinhos, Portugal; phone: +351 917069106; e-mail: joanalferreira@gmail.com. 
Cartwright and Anastasopoulou 2020). This may be due to the lack of well-defined diagnostic criteria, different management approaches to HBS, and distinct timings of diagnosis and treatment of hyperparathyroidism (Goldfarb et al. 2012; Cartwright and Anastasopoulou 2020). In the first large case series, it has been estimated that HBS developed in up to $13 \%$ of patients with PHP (Anderberg et al. 1981; Zamboni et al. 1986; Brasier and Nussbaum 1988), while in the recent case series in Asian populations the prevalence rates ranged from 24 to $87 \%$ (Agarwal et al. 2002; Bhansali et al. 2005; Gopal et al. 2010; Pradeep et al. 2011) and was only 4\% in a case series in Saudi Arabia (Malabu and Founda 2007). Regarding the patients with SHP-CKD, who underwent parathyroidectomy, HBS reports from Europe, North America, and Asia ranged from 27 to 51\% (Goldfarb et al. 2012; Latus et al. 2013; Ho et al. 2017).

The most consensual definition of HBS is a severe hypocalcemia [total serum calcium $<8.4 \mathrm{mg} / \mathrm{dL}$ or ionized calcium (iCa) $<1.12 \mathrm{mmol} / \mathrm{L}$ ] that persists for at least three days after parathyroidectomy (Brasier and Nussbaum 1988; Witteveen et al. 2013; Ho et al. 2017; Anwar et al. 2018). Serum phosphate levels decrease and persist low and parathyroid hormone (PTH) levels are normal or high (Brasier and Nussbaum 1988; Witteveen et al. 2013; Cartwright and Anastasopoulou 2020), which allow to distinguish between physiological (transient) hypocalcemia and hypocalcemia due to primary hypoparathyroidism. In addition, postoperatively serum alkaline phosphatase (ALP) levels can increase and hypomagnesemia can occur (Brasier and Nussbaum 1988; Witteveen et al. 2013). Symptoms of hypocalcemia and intravenous calcium requirement are also parameters defined as possible diagnostic criteria (Goldfarb et al. 2012; Latus et al. 2013).

HBS presumably occurs in the context of the sudden drop in PTH levels after parathyroidectomy, which leads to an abrupt and pronounced shift of bone turnover towards the osteoblast-mediated bone formation with increased influx of calcium into the bone and remineralization (Goldfarb et al. 2012; Witteveen et al. 2013).

The risk factors for the development of HBS are still debated in current literature, namely high preoperative levels of ALP, PTH and calcium, radiological evidence of bone disease, larger volume or weight of excised pathological parathyroid glands, vitamin D deficiency and advanced age (Brasier and Nussbaum 1988; Witteveen et al. 2013; Florescu et al. 2014; Anwar et al. 2018; Cartwright Anastasopoulou 2020).
The aim of this study was to characterize and compare the postoperative outcomes of patients with PHP and SHP-CKD who developed HBS in our health care center in two different time frames: before and after the implementation of a standardized protocol which defines the criteria for an intensive electrolytic monitoring and supplementation. Additionally, we also intended to compare the postoperative results between the two periods in the entire sample regardless of the HBS development.

\section{Materials and methods}

Study design. We conducted a longitudinal study in a Portuguese health care institution from July 2013 to June 2020. Our protocol was implemented in April 2017 and the patients were compared according to the time of the protocol implementation (pre- and postprotocol periods) and according to the etiology of the hyperparathyroidism (PHP group and SHP-CKD group).

Cases of familial hypocalciuric hypercalcemia, hyperparathyroidism due to other than PHP and CKD, pediatric age and pregnant women were considered exclusion criteria. Tertiary hyperparathyroidism in the context of CKD, although an entity different from SHP-CKD, was also included in our study.

This study complies with the ethical standards as laid down in the 1964 Declaration of Helsinki and its later amendments and its publication was approved by the institutional Ethical Committee.

Study criteria. Parathyroid surgery was performed by few dedicated endocrine surgeons. Surgical success of parathyroidectomy or biochemical cure was based on the "Miami" criteria developed by Irvin et al. (1991) and was defined as the reduction of intraoperative PTH levels of at least 50\% after the excision. Real time intraoperative assay of PTH serum levels were collected at baseline (at the beginning of surgery) and 10-20 min after the parathyroid gland excision. In case of multiple removal of parathyroid glands, it was performed after the removal of the last one. In the absence of surgical success, an additional parathyroid gland exploration was performed with removal of enlarged glands and new intraoperative assay of PTH level. Decline rate of intraoperative PTH levels was calculated as the ratio of PTH level at 10-20 min and baseline.

HBS was considered in cases of severe postoperative hypocalcemia (iCa $<1.12 \mathrm{mmol} / \mathrm{L}$ ) lasting at least for three days with the need for intravenous calcium supplementation during hospitalization, associated with a drop in phosphate and normal PTH levels. 
Calcium replacement was performed intravenously with calcium gluconate (infusion or bolus) and orally with calcium carbonate. Hospital readmission during the first year after the discharge was evaluated for each patient.

Literature has no universal criteria or a gold standard test to distinguish between proliferative lesions of parathyroid glands. Adenomas usually involve one single gland and parathyroid hyperplasia generally involves all glands, albeit unevenly (Shakeel et al. 2016). Our methodology was to quantify the glandular involvement (based on the number of glands with high sestamibi uptake and/or pathological signs of adenoma or hyperplasia) instead of discriminating between adenoma and hyperplasia, which was in fact ambiguous in many cases.

Protocol implementation. In the pre-protocol era, a baseline study with serum ionized calcium, PTH level and kidney function was generally performed.
Admission to Intermediate Care Unit (ICU) occurred only in cases of a specifical referral by the Assistant physician or Anesthesiologist considering the assessment of surgical risk. In the ICU, phosphocalcium monitoring was generally performed daily until transferring to the surgical ward. Patients admitted to the surgical ward after parathyroidectomy systematically started on intravenous calcium supplementation, regardless of the serum calcium levels. Analytical studies were usually performed after surgery and before discharge and additionally in case of symptoms.

In April 2017, we developed and implemented an institutional protocol, taking a multidisciplinary approach to improve health care quality and, in particular, standardizing diagnostic and therapeutic procedures. The protocol was based on the clinical practice and review of the literature. It included guidance on appropriate pre-surgical investigation

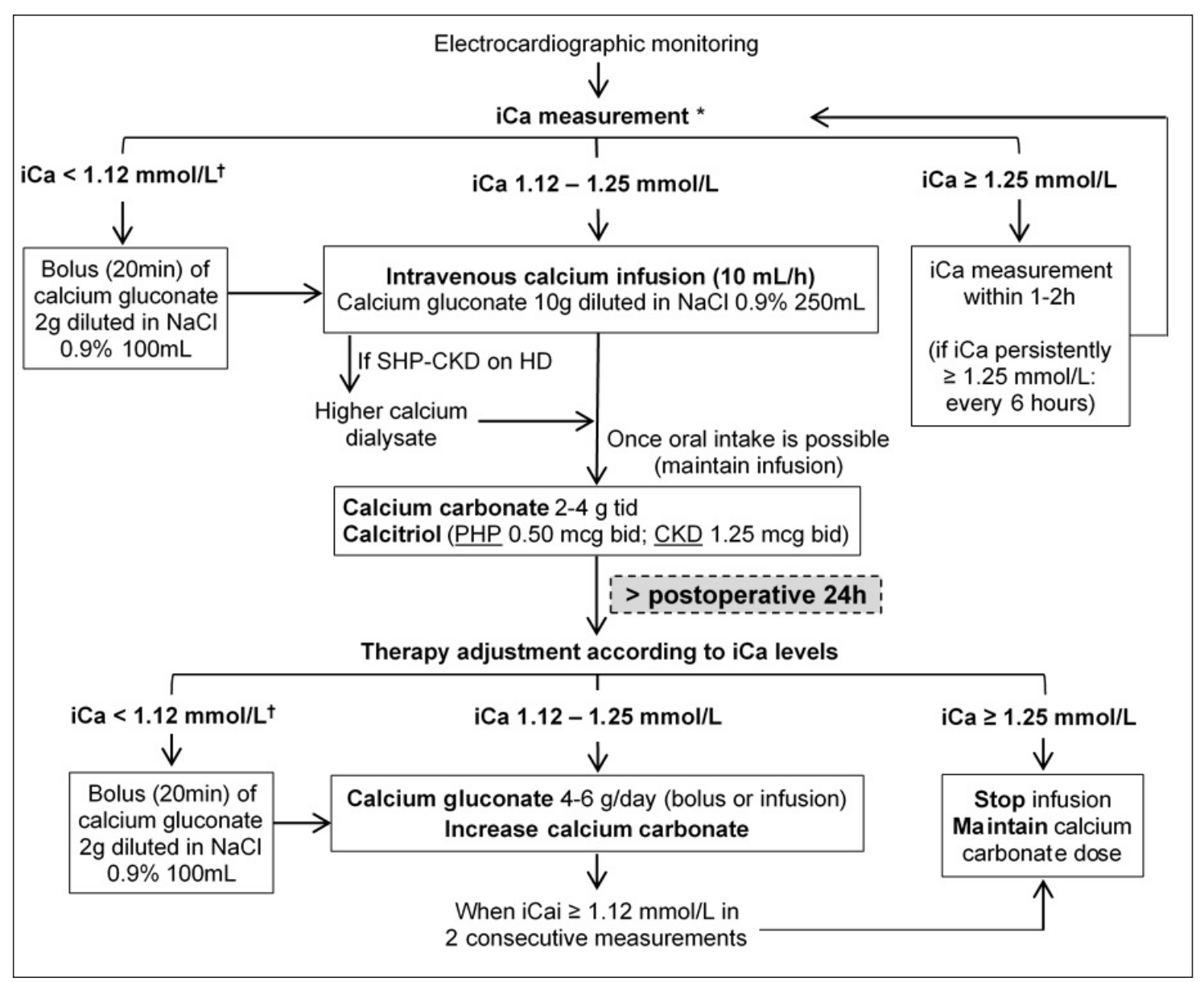

Figure. 1. Postoperative management in the ICU. Legend: ${ }^{\star}$ In the first postoperative $48 \mathrm{~h}$, ionized calcium (iCa) measurement occurs every $6 \mathrm{~h}$ and phosphate, magnesium and potassium measurements occur every $12 \mathrm{~h}$; ${ }^{\dagger}$ or symptoms of hypocalcemia; HD - hemodialysis. 
(analytic and imaging study), analytic measurements in anesthetic induction, assessment of intraoperative $\mathrm{PTH}$, and management after parathyroidectomy. In the post-protocol era, patients were promptly referred for postoperative admission in the ICU, considering the surgical risk or being at high risk for HBS: 1) severe hyperparathyroidism (preoperative PTH level above $800 \mathrm{pg} / \mathrm{mL}$, for more than one year, parathyroid volume higher than $\left.1 \mathrm{~cm}^{3}\right), 2$ ) biochemical and/ or radiological evidence of bone disease (preoperative elevated ALT levels, bone fractures, evident low bone mineral density (BMD), subperiosteal erosions), 3) vitamin D deficiency and 4) stages 4 and 5 CKD with or without dialysis (Kaya et al. 2016). Post-operative iCa levels below $1.12 \mathrm{mmol} / \mathrm{L}$ or hypocalcemic symptoms were also criteria for transfer to the ICU.

In addition to the integration of clinical and analytical data, our protocol post-surgical management in ICU includes an algorithm (Figure 1), which defines the need for electrolytic and calcitriol supplementation (the dose of the latter depends on the etiology of hyperparathyroidism). Electrolytic measurements in ICU included iCa levels every $6 \mathrm{~h}$ and phosphate and magnesium every $12 \mathrm{~h}$, up to $48 \mathrm{~h}$ after surgery. When intravenous calcium infusion was not required for $\geq 24 \mathrm{~h}$, patients were transferred to the surgical ward where iCa and phosphate were measured every day until discharge. Management in the surgical ward is provided in Figure 2. Patients were discharged when calcium levels were stable and normal for at least 24 $\mathrm{h}$ after intravenous calcium suspension. Subsequent outpatient management was done at the follow-up visit, usually within 30 days after surgery in PHP patients, and weekly (or more frequently if clinically indicated) in the case of SHP-CKD on dialysis.
Clinical and laboratory assessments. Data collection included patient's characteristics and postoperative evolution. Total amount of intravenous or oral calcium supplementation was expressed in grams (g). Duration of calcium supplementation, hypocalcemia, and hospitalization were expressed in days. Time of occurrence of hypocalcemia was expressed in hours after parathyroidectomy.

Data on endocrine assessments were retrieved from laboratory records before, during, and after parathyroidectomy. All measurements were performed in the institution laboratory. Measurement of intact PTH and 25-hydroxivitamin D [25(OH)D] were performed using chemiluminescent bridging immunoassay (CLIA) (Architect I2000sr). Laboratory reference intervals and units of each analyte were as follows: intact PTH: $15-68.3 \mathrm{pg} / \mathrm{mL}$; iCa $1.15-1.35 \mathrm{mmol} / \mathrm{L}$, phosphorus: $2.3-4.7 \mathrm{mg} / \mathrm{dL}$, magnesium: 1.6-2.6 mg/dL, ALP: 40-150 U/L. Vitamin D deficiency was considered as $25(\mathrm{OH}) \mathrm{D}$ below $20 \mathrm{ng} / \mathrm{mL}$. Stages for CKD were defined according to current KDOQI guidelines (National Kidney Foundation 2002).

Statistical analysis. Continuous variables were expressed as mean \pm standard deviation (SD), if normally distributed or median and interquartile range (IQR), if otherwise. Categorical variables were expressed as frequencies and percentages. For comparison between two groups, Student's $t$-test and Mann-Whitney $U$ test were used to compare two groups for parametric and non-parametric continuous variables, respectively. Fisher's or Chi-square tests were used to study differences between categorical variables. All analyses were performed using IBM-SPSS version 25 and the level of significance $\alpha=0.05$ was noted for all.

\begin{tabular}{|c|c|c|c|c|c|}
\hline \multirow{2}{*}{ Origin } & \multirow{2}{*}{$\begin{array}{c}\text { Calcium } \\
\text { therapy }\end{array}$} & \multicolumn{4}{|c|}{ Daily iCa levels } \\
\cline { 3 - 6 } Recovery & None & $\begin{array}{c}\text { Bolus of calcium } \\
\text { gluconate } 1 \mathrm{~g} \text { and } \\
\text { room }\end{array}$ & $\begin{array}{c}\text { Bansfer to ICU } \\
\text { Bolus of calcium } \\
\text { gluconate } 1 \mathrm{~g} \text { and } \\
\text { consider to } \\
\text { transfer to ICU }\end{array}$ & $\begin{array}{c}\text { Bolus of calcium } \\
\text { gluconate } 1 \mathrm{~g} \text { q6h } \\
\text { and start calcium } \\
\text { carbonate and } \\
\text { calcitriol per os asap }\end{array}$ & $\begin{array}{c}\text { Repeat iCa } \\
\text { measurement } \\
\text { (q6h in the first day } \\
\text { if } \geq 1.25 \text { mmol/L) }\end{array}$ \\
\hline \multirow{2}{*}{ ICU } & $\begin{array}{c}\text { Under iv } \\
\text { calcium }\end{array}$ & $\begin{array}{c}\text { Bolus of calcium } \\
\text { gluconate } 1 \mathrm{~g} \text { and } \\
\text { increase calcium } \\
\text { carbonate per os }\end{array}$ & $\begin{array}{c}\text { Reduce calcium } \\
\text { bolus and } \\
\text { increase calcium } \\
\text { carbonate per os }\end{array}$ & $\begin{array}{c}\text { Stop bolus and } \\
\text { increase calcium } \\
\text { carbonate per os }\end{array}$ & $\begin{array}{c}\text { Stop bolus and } \\
\text { maintain calcium } \\
\text { carbonate per os }\end{array}$ \\
\cline { 3 - 6 } & $\begin{array}{c}\text { Only } \\
\text { calcium } \\
\text { per os }\end{array}$ & $\begin{array}{c}\text { Bolus of calcium } \\
\text { gluconate } 1 \mathrm{~g} \text { and } \\
\text { increase calcium } \\
\text { carbonate per os }\end{array}$ & $\begin{array}{c}\text { Increase calcium } \\
\text { carbonate per os }\end{array}$ & $\begin{array}{c}\text { Maintain calcium } \\
\text { carbonate per os }\end{array}$ & $\begin{array}{c}\text { Reduce calcium } \\
\text { carbonate per os }\end{array}$ \\
\hline
\end{tabular}

Figure 2. Postoperative management in the surgical ward. Legend: ${ }^{\star}$ If ionized calcium $(\mathrm{iCa})>1$ $\mathrm{mmol} / \mathrm{L}$ for $48 \mathrm{~h}$, stop bolus and increase calcium carbonate; asap - as soon as possible; ICU Intermediate Care Unit; i.v. - intravenous; q6h - every 6 h; ${ }^{\dagger}$ or symptoms of hypocalcemia. 


\section{Results}

Patient baseline characteristics. We evaluated the records of 77 parathyroidectomies performed in 73 patients in seven years: 40 parathyroidectomies in 39 patients prior to protocol implementation and 37 parathyroidectomies in 34 patients in the postprotocol period. Overall, 61 (83.5\%) patients had PHP and 12 (16.4\%) had SHP-CKD. In SHP-CKD, $80 \%$ and $100 \%$ of the patients were on dialysis for a median duration of 7.0 and 5.3 years before and after the protocol implementation, respectively. The mean age was $63.8 \pm 14.5$ years old with a predominance of female gender (69.9\%).

Characterization and management of hyperparathyroidism. Table 1 shows the laboratorial parameters and surgical characteristics according to the etiology of the hyperparathyroidism in the periods before and after protocol implementation.

Preoperative management. Patients with PHP and SHP-CKD had similar median preoperative iCa and $\mathrm{PTH}$ levels in the periods before and after protocol implementation. In the pre-protocol era, patients did not have a standardized preoperative laboratory evaluation, namely vitamin $\mathrm{D}$, magnesium, and ALP levels. Vitamin D deficiency was detected in all studied patients with SHP-CKD and in 75.0\% with PHP (however, it was poorly screened in the latter in the pre-protocol era). In both periods, patients with detected vitamin D deficiency started on supplementation. Although there was no known case of severe adynamic bone disease, high ALP levels were detected in $11.1 \%$ in PHP and in $75.0 \%$ in SHP-CKD.

Bone densitometry was performed more often since the implementation of the protocol $(38.5 \%$ vs. $82.4 \%, \mathrm{p}<0.001)$. In the post-protocol era, low BMD (osteopenia and osteoporosis) was detected in $88.9 \%$ $(n=24)$ of patients with PHP and in the only patient with SHP-CKD tested.

Single-gland disease predominated in PHP $(79.7 \%)$, while multiple gland disease occurred in $92.3 \%$ of patients with SHP-CKD: $61.5 \%$ of this group required removal of three and often part of the fourth gland. The remaining patients had one or two parathyroid glands excised.

Intraoperative and postoperative management. Intraoperative PTH levels' decline and surgical success were similar between both periods in PHP and SHP-CKD groups. The lowest postoperative calcium levels were lower in patients with PHP in the post-protocol era, compared to the previous period (1.26 vs. $1.19 \mathrm{mmol} / \mathrm{L}, \mathrm{p}=0.020)$. It was similar in patients with SHP-CKD between the two periods.
Overall, the need for admission to the ICU was higher since the implementation of the protocol compared to the previous period $(25.0 \%$ vs. $48.6 \%$, $\mathrm{p}=0.031$ ). In the post-protocol period, $44.1 \%$ versus no patients with PHP were admitted to the ICU $(\mathrm{p}<0.001)$. PHP group had also an increased length of hospital stay ( 2.0 vs. 3.0 days, $\mathrm{p}=0.017)$. In both periods, all patients with SHP-CKD were admitted in ICU. No differences were found between the total and ICU hospital stays in this group.

Before protocol implementation, $86.7 \%$ of patients with PHP and $90.0 \%$ with SHP-CKD were supplemented with intravenous calcium in the first 24 hours after surgery. In the post-protocol era, 76.5\% of patients with PHP and all patients with SHP-CKD required intravenous calcium gluconate supplementation. The latter required a lower time on intravenous calcium than before ( 3 days vs. 1 day, $\mathrm{p}=0.010$ ). Subsequent oral calcium use was the rule for almost all patients with SHP-CKD, but in the PHP group a higher need of these supplements in the second period was noted ( $20.0 \%$ vs. $50.0 \%, \mathrm{p}=0.013)$. According to the protocol, calcitriol was prescribed in all patients admitted to the ICU after parathyroidectomy.

The median preoperative phosphorus levels were $2.3 \mathrm{mg} / \mathrm{dL}$ in the PHP group, and $4.5 \mathrm{mg} / \mathrm{dL}$ in the SHP-CKD group. After parathyroidectomy, the median phosphorus levels changed to $3.5 \mathrm{mg} / \mathrm{dL}$ in the PHP group, and $2.1 \mathrm{mg} / \mathrm{dL}$ in the SHP-CKD group. Some degree of postoperative decrease in phosphorus levels was verified in $40.0 \%(n=8)$ of patients with PHP and in $66.7 \%(n=2)$ of patients with SHP-CKD. In the post-protocol era, the median pre- and postoperative magnesium levels were 2.02 and $1.75 \mathrm{mg} / \mathrm{dL}$ in patients with PHP, and 2.00 and $2.08 \mathrm{mg} / \mathrm{dL}$ in patients with SHP-CKD, respectively. Some degree of postoperative decrease in magnesium levels was noted in $85.0 \%(n=17)$ of patients with PHP and in $33.3 \%(n=1)$ of patients with SHP-CKD. In the cases of magnesium or phosphorus levels below the lower limit of normal, supplementation was started.

Cases of hungry bone syndrome. Of the 77 parathyroidectomies, a total of 13 patients presented HBS in seven years with an overall prevalence of $16.9 \%$ and an incidence of 1.9 cases per year in our institution. Table 2 shows the characterization of all the patients who experienced HBS. In the pre-protocol period, all four patients tested had vitamin $\mathrm{D}$ deficiency, three of them with SHP-CKD. Regarding the post-protocol era, all patients were tested for vitamin $\mathrm{D}$ status and its deficiency was observed in $80.0 \%(n=4)$ of patients with PHP and in the two patients with SHP-CKD. In the post-protocol era, low BMD was detected in 
Table 1

Laboratorial parameters and surgical characteristics regarding the etiology of the hyperparathyroidism before and since protocol implementation.

\begin{tabular}{|c|c|c|c|c|c|c|}
\hline \multirow{2}{*}{ Parameters } & \multicolumn{3}{|c|}{ PHP group } & \multicolumn{3}{|c|}{ SHP-CKD group } \\
\hline & Pre-protocol & Post-protocol & p-value & Pre-protocol & Post-protocol & p-value \\
\hline Parathyroidectomy [n (\%)] & $30(46.9)$ & $34(53.1)$ & - & $10(76.9)$ & $3(23.1)$ & - \\
\hline Age at first surgery (mean $\pm S D)$ & $63.1 \pm 15.6$ & $65.2 \pm 13.4$ & 0.577 & $61.3 \pm 16.9$ & $63.0 \pm 5.7$ & 0.894 \\
\hline Female gender $[\mathbf{n}(\%)]$ & $21(72.4)$ & $23(71.9)$ & 0.963 & $6(60.0)$ & $1(50.0)$ & 1.000 \\
\hline \multicolumn{7}{|l|}{ Preoperative period } \\
\hline iCa levels [median (IQR)] & $1.56(0.22)$ & $1.53(0.16)$ & 0.214 & $1.41(0.43)$ & $1.43(-)$ & 0.858 \\
\hline Highest PTH levels [median (IQR)] & $226.8(378.3)$ & $157.3(138.3)$ & 0.186 & $721.6(1142.4)$ & $1626.0(-)$ & 0.087 \\
\hline Phosphorus levels [median (IQR)]] & $2.5(0.8)$ & $2.3(0.5)$ & 0.625 & $4.4(0.6)$ & $4.5(-)$ & 0.813 \\
\hline Magnesium levels [median (IQR)] & - & $2.0(0.3)$ & - & - & $1.9(-)$ & - \\
\hline Vitamin D deficiency [n (\%)] & $7(87.5) \mathrm{a}$ & $23(71.9)$ & - & $1(100.0) b$ & $3(100.0)$ & - \\
\hline High ALP levels [n (\%)] & $2(25.0) c$ & $2(7.1)$ & 0.207 & $1(50.0) \mathrm{d}$ & $2(100.0)$ & 1.000 \\
\hline One excised parathyroid gland [n (\%)] & $18(60.0)$ & $23(67.6)$ & 0.525 & $1(10.0)$ & $2(66.7)$ & 0.108 \\
\hline Single-gland involvement [n (\%)] & $23(79.3)$ & $28(82.4)$ & 0.755 & $1(10.0)$ & $0(0.0)$ & 1.000 \\
\hline \multicolumn{7}{|l|}{ Intraoperative PTH levels } \\
\hline Decline rate [median (IQR)] & $74.3(13.1)$ & $81.1(19.8)$ & 0.160 & $79.0(26.8)$ & $62.4(-)$ & 0.233 \\
\hline Surgical success rate $[\mathrm{n}(\%)]$ & $28(93.3)$ & $30(88.2)$ & 0.676 & $8(80.0)$ & $2(66.7)$ & 1.000 \\
\hline Decline rate of PTH [median (IQR)] & $75.2(12.4)$ & $81.4(15.8)$ & 0.080 & $79.4(7.4)$ & $70.6(-)$ & 0.190 \\
\hline \multicolumn{7}{|l|}{ Postoperative period } \\
\hline Lowest iCa levels [median (IQR)] & $1.26(0.11)$ & $1.19(0.14)$ & $0.020^{*}$ & $1.08(0.33)$ & $1.11(-)$ & 0.644 \\
\hline Symptoms of hypocalcemia [n (\%)] & $2(6.6)$ & $3(8.8)$ & 0.745 & $5(50.0)$ & $0(0.0)$ & 0.231 \\
\hline \multicolumn{7}{|l|}{ Intravenous calcium supplementation } \\
\hline Patients $[\mathrm{n}(\%)]$ & $26(86.7)$ & $26(76.5)$ & 0.351 & $9(90.0)$ & $3(100.0)$ & 1.000 \\
\hline Duration [median (IQR)] & $1.0(1.0)$ & $1.0(1.0)$ & 0.407 & $3.0(4.0)$ & $1.0(0.0)$ & $0.010^{*}$ \\
\hline Total amount [median (IQR)] & $5.0(5.0)$ & $4.5(6.3)$ & 0.424 & $12.0(18.0)$ & $10.0(-)$ & 0.224 \\
\hline \multicolumn{7}{|l|}{ Oral calcium supplementation } \\
\hline Patients [n (\%)] & $6(20.0)$ & $17(50.0)$ & $0.013^{*}$ & $9(90.0)$ & $3.0(100.0)$ & 1.000 \\
\hline Duration [median (IQR)] & $2.5(4.0)$ & $5.0(3.0)$ & 0.367 & $5.0(5.0)$ & $5.0(-)$ & 0.638 \\
\hline Total amount [median (IQR)] & $6.3(22.9)$ & $17.5(18.9)$ & 0.074 & $23.0(39.0)$ & $27.0(-)$ & 0.853 \\
\hline \multicolumn{7}{|l|}{ ICU stay } \\
\hline Patients [n (\%)] & $0(0.0)$ & $15(44.1)$ & $<0.001^{\star}$ & $10(100.0)$ & $3(100.0)$ & - \\
\hline Length of stay [median (IQR)] & - & $3.0(1.0)$ & - & $3.5(3.0)$ & $3.0(-)$ & 0.795 \\
\hline Length of hospital stay [median (IQR)] & $2.0(2.0)$ & $3.0(4.0)$ & $0.017^{*}$ & $10.0(5.0)$ & $7.0(-)$ & 0.122 \\
\hline
\end{tabular}

${ }^{\star}$ Statistically significant; $a-8$ patients ( $73.3 \%$ of missing values); $b-1$ patient ( $90 \%$ of missing values); $\mathrm{c}-8$ patients ( $73.3 \%$ of missing values); $\mathrm{d}-8$ patients ( $80 \%$ of missing values); $\mathrm{e}-16$ patients with more than one postoperative iCa (55.2\% of missing values); $\mathrm{f}-6$ patients with more than one postoperative $\mathrm{iCa}$ ( $40.0 \%$ of missing values).

$80.0 \%(n=4)$ of the HBS cases with PHP and in the only patient with SHP-CKD tested. Of the patients with PHP and low BMD, 16.7\% $(n=4)$ developed HBS.

In both periods, most patients reached iCa levels much lower than $1.12 \mathrm{mmol} / \mathrm{L}$, even with calcium and calcitriol supplementation. In many cases, espe- cially in the pre-protocol era, diagnosis of HBS was not recognized during the treatment of patients.

Comparison of HBS cases between the two periods is shown in Table 3. The prevalence of HBS was similar between the two periods: $15.0 \%(n=6)$ in the first period and $18.9 \%(n=7)$ since protocol imple- 


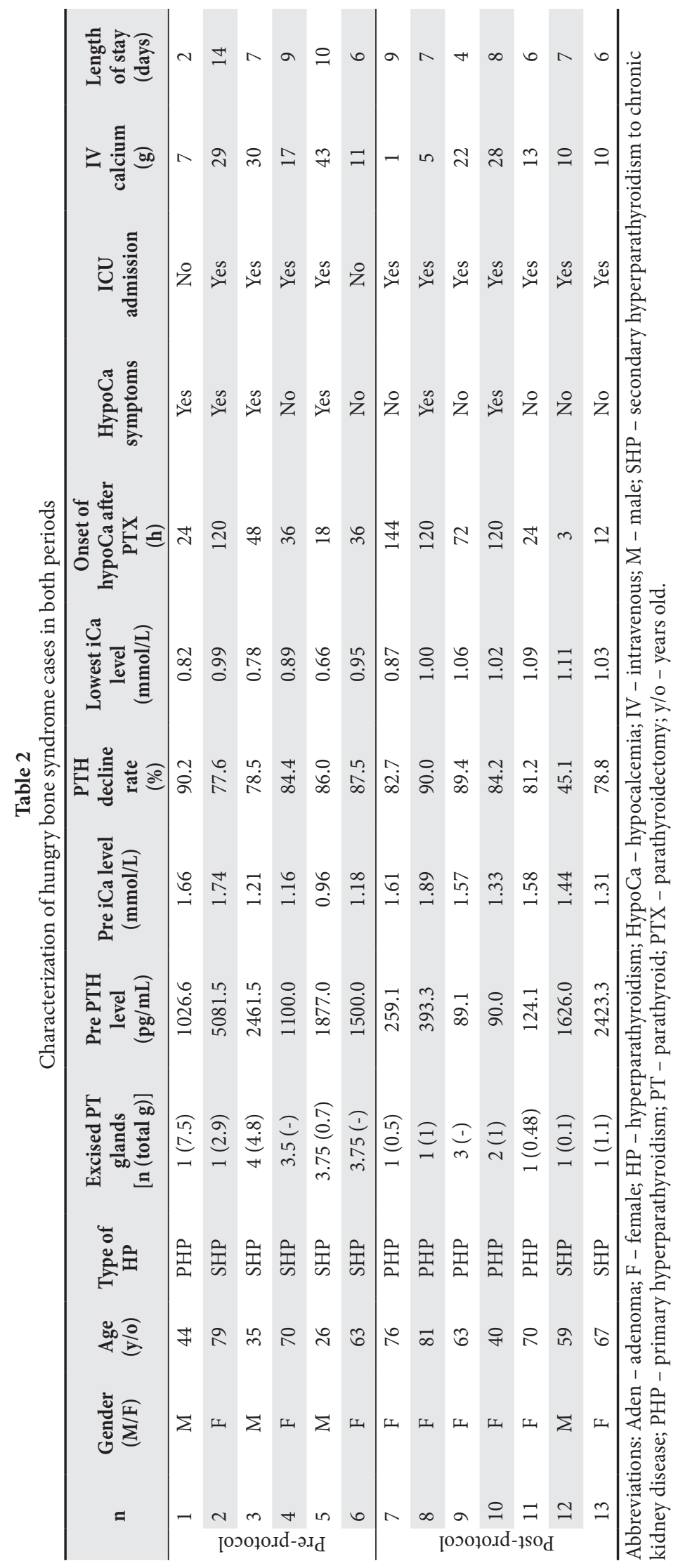

mentation $(\mathrm{p}=0.646)$. The prevalence of HBS in patients with PHP increased from $3.3 \%$ in pre-protocol period to $14.7 \%$ since its implementation, but with no statistical significance $(\mathrm{p}=0.202)$. Overall, patients with SHP-CKD had a higher rate of HBS compared to PHP etiology [58.3\% ( $n=7)$ vs. $9.8 \%(\mathrm{n}=6), \mathrm{p}<0.001]$.

The first recorded evidence of hypocalcemia in patients with HBS varied significantly between the two periods. In the preprotocol period, $66.7 \%$ of the patients had symptoms in the first place (paresthesia in extremities and perioral region and muscle cramps). No arrhythmia, convulsive episodes or laryngospasm were described. In the post-protocol era, all patients were diagnosed by blood tests $(\mathrm{p}=0.021)$. No electrocardiographic alterations were detected in these cases. After the detection of hypocalcemia, two patients had paresthesia in the extremities. In the pre-protocol era, patients experienced a significantly higher duration of hypocalcemia (4 vs. 2 days, $\mathrm{p}=0.047)$ and had a higher overall rate of hypocalcemia symptoms, however without statistical significance $(66.6 \%$ vs. $28.6 \%$, $\mathrm{p}=0.286$ ).

A postoperative decrease in phosphorus and magnesium levels were noted within the first five days for PHP and within the first two days for SHP. The median preand post-operative levels of phosphorus were 3.8 and $3.0 \mathrm{mg} / \mathrm{dL}$ in patients with PHP, and 4.5 and $2.0 \mathrm{mg} / \mathrm{dL}$ in patients with SHP-CKD, respectively. In the postprotocol era, the median pre- and postoperative magnesium levels were 1.93 and $1.65 \mathrm{mg} / \mathrm{dL}$ in patients with PHP, and 1.92 and $1.96 \mathrm{mg} / \mathrm{dL}$ in patients with SHP-CKD, respectively.

In the pre-protocol era, all patients with HBS initiated intravenous calcium supplementation in the first $24 \mathrm{~h}$ after parathyroidectomy, while about half of the patients did it in the second period (100\% vs. $57.1 \%$, $\mathrm{p}=0.192)$. The median duration and total amount of intravenous calcium were also tending to be higher in the first period compared to the second one (5.5 vs. 2.0 days, $\mathrm{p}=0.083$, and 23.0 vs. $10.0 \mathrm{~g}, \mathrm{p}=0.116$, respectively). Regarding oral calcium, no differences were found between the two 
periods. All patients with HBS were indicated to continue with oral calcium supplements at time of hospital discharge.

In the post-protocol era, all patients who developed HBS were admitted to the ICU, unlike the previous period. The length of hospital stay was similar between the two periods ( 9.5 vs. 7.0 days, $p=0.281$ ), while the time spent in the surgical ward was significantly lower since the protocol was implemented (7.0 vs. 3.0 days, $\mathrm{p}=0.042$ ). Readmission due to hypocalcemia was needed in two patients in the pre-protocol era and in none after that.

\section{Discussion}

In our study, the implementation of the protocol led to the tendency of improved detection and treatment of HBS in patients with PHP. None of the diagnosis of HBS was established due to hypocalcemic symptoms in the post-protocol era (contrary to preprotocol period). Both hypocalcemia length and duration of surgical ward hospitalization were reduced. Additionally, the protocol definitely improved the assessment of the totality of patients with PHP or SHP-CKD before and after parathyroidectomy. In

Table 3

Comparison of hungry bone syndrome cases between the two periods regarding protocol implementation.

\begin{tabular}{|c|c|c|c|}
\hline Parameters & Pre-protocol & Post-protocol & p-value \\
\hline Patients with HBS [n (\%)] & $6(15.0)$ & $7(18.9)$ & 0.646 \\
\hline PHP $[\mathrm{n}(\%)]$ & $1(3.3)$ & $5(14.7)$ & 0.202 \\
\hline SHP-CKD [n (\%)] & $5(50.0)$ & $2(66.7)$ & 1.000 \\
\hline Age at first surgery [median (IQR)] & $53.5(40)$ & $67.0(17)$ & 0.316 \\
\hline Female gender $[\mathbf{n}(\%)]$ & $3(50.0)$ & $6(85.7)$ & 0.266 \\
\hline \multicolumn{4}{|l|}{ First evidence of hypocalcemia } \\
\hline Symptoms $[\mathrm{n}(\%)]$ & $4(66.7)$ & $0(0.0)$ & $0.021^{*}$ \\
\hline Analytic hypocalcemia [n (\%)] & $2(33.3)$ & $7(100.0)$ & \\
\hline \multicolumn{4}{|l|}{ Lowest postoperative iCa } \\
\hline iCa level [median (IQR)g & $0.92(0.28)$ & $1.03(0.09)$ & 0.116 \\
\hline Time of occurrence [median (IQR)] & $36(44)$ & $72(108)$ & 0.719 \\
\hline \multicolumn{4}{|l|}{ Hypocalcemia } \\
\hline Duration [median (IQR)] & $6(10)$ & $4(1)$ & $0.041^{*}$ \\
\hline Symptoms $[\mathrm{n}(\%)]$ & $4(66.6)$ & $2(28.6)$ & 0.286 \\
\hline \multicolumn{4}{|l|}{ Intravenous calcium supplementation } \\
\hline Initiation <24 h after surgery [n (\%)] & $6(100.0)$ & $4(57.1)$ & 0.192 \\
\hline Total duration [median (IQR)] & $5.5(5.0)$ & $2.0(4.0)$ & 0.083 \\
\hline Total amount [median (IQR)] & $23.0(23.0)$ & $10.0(17.0)$ & 0.116 \\
\hline \multicolumn{4}{|l|}{ Oral calcium supplementation } \\
\hline Initiation $<24 \mathrm{~h}$ after surgery [n (\%)] & $1(20.0)$ & $2(28.6)$ & 1.000 \\
\hline Total duration [median (IQR)] & $5.0(4.0)$ & $6.0(4.0)$ & 0.739 \\
\hline Total amount [median (IQR)] & $61.5(50.5)$ & $27.0(38.5)$ & 0.514 \\
\hline \multicolumn{4}{|l|}{ Place of hospitalization } \\
\hline ICU $[\mathrm{n}(\%)]$ & $4(66.7)$ & $7(100.0)$ & 0.192 \\
\hline Surgical ward [n (\%)] & $6(100.0)$ & $7(100.0)$ & - \\
\hline Length of hospital stay [median (IQR)] & $9.5(7.0)$ & $7.0(2.0)$ & 0.281 \\
\hline ICU [median (IQR)] & $1.0(4.0)$ & $4.0(1.0)$ & 0.110 \\
\hline Surgical ward [median (IQR)] & $7.0(4.0)$ & $3.0(1.0)$ & $0.042^{*}$ \\
\hline Readmission due to hypocalcemia [n (\%)] & $2(33.3)$ & $0(0.0)$ & 0.192 \\
\hline
\end{tabular}

*Statistically significant 
patients with PHP, intensive monitoring of phosphocalcium metabolism after parathyroidectomy allowed to detect lower calcium levels compared to the preprotocol era, when they were undoubtedly underdiagnosed. It also led to a significant increase in postoperative oral calcium replacement and the admission to the ICU of high-risk patients for intensive electrolyte monitoring (which did not occur previously). In patients with SHP-CKD, a significantly shorter duration of intravenous calcium replacement and a trend towards a reduction in the total length of hospital stay were noted after the implementation of the protocol.

To the authors knowledge, there are no controlled studies regarding the management of patients with HBS. Regarding the lack of prospective data, HBS studies remain scarce in the general population. In the present study, the overall prevalence of HBS was 9.8\% in PHP and 58.3\% in SHP-CKD. The prevalence of 3.3\% in patients with PHP in the pre-protocol era was probably underestimated, considering the higher rates reported in the literature and also in our institution since the implementation of the protocol, which is similar to other studies (Anderberg et al. 1981; Zamboni et al. 1986; Brasier and Nussbaum 1988). The low prevalence of HBS reported in Saudi Arabia (Malabu and Founda 2007) may likely be due to the retrospective data collection and possibly of a high amount of missing data. In our study, the establishment of an algorithm of management and timings of postoperative blood tests allowed a prospectively collection of data and a substantial improvement of data quality in the post-protocol era. Probably, some patients with PHP in the pre-protocol era have experienced HBS, but it was not detected, even in this retrospective analysis. The intensive monitoring and the consideration on the need for ICU admission defined by the protocol have possibly conducted to the diagnosis of hidden cases of HBS in patients with PHP, who were discharged earlier in the preprotocol era. Regarding the SHP-CKD etiology, the rate of HBS was quite similar to the literature, namely with those reported in Germany (Latus et al. 2013). In fact, both in the pre- and post-protocol periods, all patients with SHP-CKD were admitted to the ICU after parathyroidectomy. This accordance with the literature further highlights the importance of a close electrolytic monitoring in the postoperative phase in patients with SHP-CKD.

This study revealed highly relevant points, despite the small sample size of patients who developed HBS. Patients managed in the pre-protocol era had a high rate of mild to moderate symptoms of hypocalcemia as the first evidence of HBS, which did not occur in the post-protocol period. In none of the periods, cardiac arrhythmia, seizures or laryngospasm due to severe hypocalcemia were recorded, which are the main concerns regarding the HBS diagnosis. In addition, patients had a significantly higher duration of hypocalcemia in the pre-protocol era. Even with prolonged hypocalcemia, some of patients remained on the surgical ward or were discharged. Hypocalcemic symptoms or readmission due to hypocalcemia were the key for the diagnosis of HBS in some patients in the pre-protocol period. In fact, a less regular phosphocalcium monitoring can significantly delay the detection of severe and persistent hypocalcemia. It is not feasible to monitor calcium levels and adapt the calcium replacement regularly when patients remain in the surgical ward, in contrary to what happens in an ICU. Therefore, patients with high-risk criteria of HBS should be admitted to the ICU for a tighter surveillance and more intensive calcium therapy to avoid persistence and aggravation of hypocalcemia. In this context, a significantly decreased length of surgical ward stay and a tendency for increased length of ICU admission were in the post-protocol era compared to the previous period. In fact, the purpose of the protocol was to ensure a timely diagnosis and treatment of HBS, as many cases were previously underdiagnosed. However, too many patients may have been admitted to the ICU in the post-protocol era. In the future, we intend to optimize this protocol for a tighter selection on admission to the ICU, but without excluding patients who may develop HBS. Another interesting finding was the tendency for shorter duration of intravenous calcium supplementation in the post-protocol period, without adverse outcomes. With the intensive electrolyte monitoring, the lowest hypocalcemia detected in patients who developed HBS tended to be higher than in the pre-protocol era. This is an indirect evidence of the improvement in the postoperative approach of these patients. Considering all these findings, we admit the occurrence of less severe cases of HBS in the post-protocol era.

In SHP-CKD, there are some postoperative management protocols reported. The general approach is to start calcium replacement before hypocalcemia occurs (Loke et al. 2009; Goh et al. 2010; Wong et al. 2020), just as in our protocol. However, calcium regimens and timings are not consensual. Loke et al. (2009) have suggested to monitor calcium levels every six hours in the first four days and published a titration regimen of intravenous calcium depending on the calcium level. Our protocol also defined this timing of calcium monitoring, while 
patients are on ICU and at least one daily blood test in the surgical ward until discharge. In fact, Cozzolino et al. (2004) have also advocated a daily follow-up of serum calcium and phosphorus. In addition, our single intravenous calcium regimen and early initiation of oral calcium intake are more convenient.

In PHP, the need for the implementation of a standardized follow-up protocol in the postoperative phase was highlighted (Oltmann et al. 2011). To the best of our knowledge, there are no protocols available regarding the HRP patients, except the studies published by Norman and colleagues (Norman and Politz 2007; Vasher et al. 2010). They have recommended an immediate discharge after parathyroidectomy and an oral calcium regimen to start within three hours after surgery, in which the maintenance dose lasts two years or more. Postoperative calcium requirements were defined according to preoperative calcium levels, BMD and finding of adenoma versus hyperplasia at parathyroidectomy. They have suggested that their protocol eliminates the development of symptomatic hypocalcemia in more than $92 \%$ of patients. However, it is dubious how the distinction between an adenoma and hyperplasia is made. In addition, the maintenance dose is too long and inconvenient for patients. Although direct comparisons with our study should be cautious, as Norman and Politz (2007) did not specifically assess the occurrence of HBS, our protocol can be an alternative in patients with PHP. In our study, we achieved an early detection of HBS (which is easily not diagnosed in PHP) and adjusted its treatment. In addition, calcium supplementation usually took only about a month without increasing the hospital readmissions.

In this study, other results may provide clues to improve our preoperative management in the future. Vitamin D deficiency was quite prevalent in patients who developed HBS and it is evoked as a risk factor for HBS (Stewart et al. 2005). Therefore, its treatment and correction before parathyroidectomy may probably reduce the occurrence of HBS, although this has not yet been proven (Witteveen et al. 2013). In addition, low BMD was also detected in most patients with HBS. However, only a portion of patients with osteopenia or osteoporosis developed HBS. Probably, the development of HBS depends on the degree of decreased BMD and increased bone turnover.
Preoperative treatment with vitamin D analogs and bisphosphonates has been advocated to decrease the severity of the postoperative hypocalcemia in patients with PHP, but more studies are needed (Witteveen et al. 2013; Mayilvaganan et al. 2017). These approaches may possibly alleviate the decline in calcium and PTH levels in the perioperative period.

Our findings show that this protocol could accomplish a more complete preoperative investigation, significantly increase the detection of HBS, and decrease the severity of these episodes (no symptoms in the time of diagnosis, minimum calcium levels not as low as previously and shorten duration of hypocalcemia). Our results support the importance of defining the criteria for patients who need close monitoring in the ICU, calcium initiation without waiting for hypocalcemia to occur, and a regular monitoring to adjust treatment and to allow a safer discharge. ICU admission may be considered in case of postoperative hypocalcemia, previously identified high risk patients of HBS or for those with high surgical risk.

A strength of this study is the inclusion of both primary and secondary etiologies of hyperparathyroidism and despite having a different pathophysiology, it allows a representative characterization of the actual clinical practice of patients who underwent parathyroidectomy. To the authors knowledge, most, if not all, articles address one of the etiologies of hyperparathyroidism. On the other hand, the small number of patients to whom HBS has developed and the discrepancy of data quality between the two periods may underestimate the impact of the implementation of the protocol.

In conclusion, we strongly recommend the implementation of a standardized protocol with an intensive monitoring of calcium levels and an algorithm regarding electrolytic supplementation as it improves the health care and the management of an eventual HBS in high-risk patients who underwent parathyroidectomy due to hyperparathyroidism.

\section{Acknowledgements}

The authors would like to thank all physicians who approached the patients with hyperparathyroidism who underwent parathyroidectomy and for following the protocol.

\section{References}

Agarwal G, Mishra SK, Kar DK, Singh AK, Arya V, Gupta SK, Mithal A. Recovery pattern of patients with osteitis fibrosa cystica in primary hyperparathyroidism after successful parathyroidectomy. Surgery 132, 1075-1083, 2002. 
Anderberg B, Gillquist J, Larsson L, Lundstrom B. Complications to subtotal parathyroidectomy. Acta Chir Scand 147, 109-113, 1981.

Anwar F, Abraham J, Nakshabandi A, Lee E. Treatment of hypocalcemia in hungry bone syndrome: A case report. Int J Surg Case Rep 51, 335-339, 2018.

Bhansali A, Masoodi SR, Reddy KS, Behera A, das Radotra B, Mittal BR, Katariya RN, Dash RJ. Primary hyperparathyroidism in north India: a description of 52 cases. Ann Saudi Med 25, 29-35, 2005.

Brasier AR, Nussbaum SR. Hungry bone syndrome: clinical and biochemical predictors of its occurrence after parathyroid surgery. Am J Med 84, 654-660, 1988.

Cartwright C, Anastasopoulou C. Hungry Bone Syndrome. In: StatPearls, Treasure Island (FL), 2020.

Cozzolino M, Gallieni M, Corsi C, Bastagli A, Brancaccio D. Management of calcium refilling post-parathyroidectomy in end-stage renal disease. J Nephrol 17, 3-8, 2004.

Florescu MC, Islam KM, Plumb TJ, Smith-Shull S, Nieman J, Mandalapu P. Calcium supplementation after parathyroidectomy in dialysis and renal transplant patients. Int J Nephrol Renovasc Dis 7, 183-190, 2014.

Goh BL, Yudisthra MG, Hisham AN. Alkaline phosphatase predicts calcium requirements after total parathyroidectomy in patients receiving dialysis. Br J Surg 97, 185-188, 2010.

Goldfarb M, Gondek SS, Lim SM, Farra JC, Nose V, Lew JI. Postoperative hungry bone syndrome in patients with secondary hyperparathyroidism of renal origin. World J Surg 36, 1314-1319, 2012.

Gopal RA, Acharya SV, Bandgar T, Menon PS, Dalvi AN, Shah NS. Clinical profile of primary hyperparathyroidism from western India: a single center experience. J Postgrad Med 56, 79-84, 2010.

Ho LY, Wong PN, Sin HK, Wong YY, Lo KC, Chan SF, Lo MW, Lo KY, Mak SK, Wong AK. Risk factors and clinical course of hungry bone syndrome after total parathyroidectomy in dialysis patients with secondary hyperparathyroidism. BMC Nephrol 18, 12, 2017.

Irvin GL, 3rd, Dembrow VD, Prudhomme DL. Operative monitoring of parathyroid gland hyperfunction. Am J Surg 162, 299-302, 1991.

Kaya C, Tam AA, Dirikoc A, Kilicyazgan A, Kilic M, Turkolmez S, Ersoy R, Cakir B. Hypocalcemia development in patients operated for primary hyperparathyroidism: Can it be predicted preoperatively? Arch Endocrinol Metab 60, 465-471, 2016.

Latus J, Roesel M, Fritz P, Braun N, Ulmer C, Steurer W, Biegger D, Alscher MD, Kimmel M. Incidence of and risk factors for hungry bone syndrome in 84 patients with secondary hyperparathyroidism. Int J Nephrol Renovasc Dis 6, 131-137, 2013.

Loke SC, Kanesvaran R, Yahya R, Fisal L, Wong TW, Loong YY. Efficacy of an intravenous calcium gluconate infusion in controlling serum calcium after parathyroidectomy for secondary hyperparathyroidism. Ann Acad Med Singapore 38, 1074-1080, 2009.

Malabu UH, Founda MA. Primary hyperparathyroidism in Saudi Arabia: a review of 46 cases. Med J Malaysia 62, 394-397, 2007.

Mayilvaganan S, Vijaya Sarathi HA, Shivaprasad C. Preoperative zoledronic acid therapy prevents hungry bone syndrome in patients with primary hyperparathyroidism. Indian J Endocrinol Metab 21, 76-79, 2017.

National Kidney Foundation. K/DOQI Clinical Practice Guidelines for Chronic Kidney Disease: evaluation, classification, and stratification. Am J Kidney Dis 39, S1-S266, 2002.

Norman JG, Politz DE. Safety of immediate discharge after parathyroidectomy: a prospective study of 3,000 consecutive patients. Endocr Pract 13, 105-113, 2007.

Oltmann SC, Maalouf NM, Holt S. Significance of elevated parathyroid hormone after parathyroidectomy for primary hyperparathyroidism. Endocr Pract 17 Suppl 1, 57-62, 2011.

Pradeep PV, Jayashree B, Mishra A, Mishra SK. Systematic review of primary hyperparathyroidism in India: the past, present, and the future trends. Int J Endocrinol 2011, 921814, 2011.

Shakeel S, Mubarak M. Proliferative Lesions of Parathyroid Glands: An Update for Practicing Pathologists. J Coll Physicians Surg Pak 26, 51-59, 2016.

Stewart ZA, Blackford A, Somervell H, Friedman K, Garrett-Mayer E, Dackiw AP, Zeiger MA. 25-hydroxyvitamin D deficiency is a risk factor for symptoms of postoperative hypocalcemia and secondary hyperparathyroidism after minimally invasive parathyroidectomy. Surgery 138, 1018-1025; discussion 1025-1026, 2005.

Vasher M, Goodman A, Politz D, Norman J. Postoperative calcium requirements in 6,000 patients undergoing outpatient parathyroidectomy: easily avoiding symptomatic hypocalcemia. J Am Coll Surg 211, 49-54, 2010.

Witteveen JE, van Thiel S, Romijn JA, Hamdy NA. Hungry bone syndrome: still a challenge in the post-operative management of primary hyperparathyroidism: a systematic review of the literature. Eur J Endocrinol 168, R45-R53, 2013. 
Wong J, Fu WH, Lim ELA, Ng CFJ, Choong HL. Hungry bone syndrome after parathyroidectomy in end-stage renal disease patients: review of an alkaline phosphatase-based treatment protocol. Int Urol Nephrol 52, 557-564, 2020.

Zamboni WA, Folse R. Adenoma weight: a predictor of transient hypocalcemia after parathyroidectomy. Am J Surg 152, 611-615, 1986. 